\title{
Аддиктология
}

\author{
УДК 616.89-02:615+616.89-008.465:614.283
}

Для цитирования: Афанасьева Н.А., Коробицина Т.В., Пичугина Ю.А., Юков О.С., Ширшова А.А. Клиникосиндромологические особенности интоксикационных психозов, вызванных употреблением современных синтетических психоактивных веществ. Сибирский вестник психиатрии и наркологии. 2021 ; 2 (111): 46-53. https://doi.org/10.26617/1810-3111-2021-2(111)-46-53

\section{Клинико-синдромологические особенности интоксикационных психозов, вызванных употреблением современных синтетических психоактивных веществ}

\section{Афанасьева Н.А.', Коробицина Т.В.' ${ }^{1}$, Пичугина Ю.А.', Юков О.С.', Ширшова A.A.'}

\author{
'ФГБОУ ВО «Красноярский государственный медицинский университет им. проф. В.Ф. Войно-Ясенеикого» \\ Минздрава России \\ Россия, 660022, Красноярск, ул. Партизана Железняка, 1 \\ ${ }^{2}$ ГАОУ ВО «Сибирский федеральный университет» \\ Россия, 660041, Красноярск, пр. Свободный, 79
}

\section{PEЗЮME}

Актуальность. Непрекращающееся производство заново синтезируемых аналогов синтетических наркотиков с немедленной их поставкой на наркорынок отражает тенденцию роста масштабов злоупотребления веществами, находящимися под международным контролем. Комплексный анализ клинической картины интоксикационных психозов и медико-социальных последствий употребления современных синтетических психоактивных веществ позволит разработать терапевтические подходы к лечению и реабилитации таких больных. Цель. Изучить особенности психозов, развившихся на фоне потребления синтетических психоактивных веществ. Материал исследования. Сформированы три группы пациентов (всего 318 мужчин, сред-

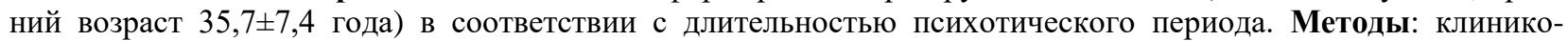
психопатологический, анамнестический, катамнестический, газожидкостная хроматография. Результаты. Получены данные об особенностях клинической картины психотических осложнений в зависимости от продолжительности психоза, а также выявления психоактивного вещества в биологических жидкостях организма пациентов. Заключение. Определены клинико-синдромологические особенности психотических расстройств, развившихся на фоне употребления новых синтетических психостимуляторов и каннабиноидов. При практически одинаковом симптомокомплексе в клинической картине таких психозов отмечены определенные отличия с учетом продолжительности психотических осложнений.

Ключевые слова: интоксикационные психозы, психоактивные вещества, шизофреноформные психические расстройства, длительность психотического периода.

\section{ВВЕДЕНИЕ}

Согласно данным последнего Всемирного доклада о наркотиках, опубликованного Управлением ООН по наркотикам и преступности (УНП $\mathrm{OOH})$, более 35 миллионов человек во всем мире страдают от расстройств, связанных с употреблением психоактивных веществ (ПАВ), и нуждаются в лечении [1]. Учитывая цикличную динамику развития и высокий уровень латентности, неблагоприятные последствия употребления наркотиков для здоровья являются более серьезными и широко представленными, чем считалось ранее [2, 3, 4, 5]. Распространение синтетических ПАВ на территории РФ привело к относительному снижению доли потребителей опиатов и в то же время к увеличению доли лиц с зависимостью от психостимуляторов и полинаркоманией. Причем более $60 \%$ больных, находящихся под диспансерным наблюдением, - люди в возрасте 18-30 лет $[5,6,7]$. На территории Красноярского края также отмечается проблема распространенности потребления современных синтетических ПАВ, что привело к значительному увеличению числа госпитализированных пациентов с психотическими расстройствами - с 2 до 577 случаев за период 2011-2014 гг. с зафиксированной тенденцией к снижению к 2018 г. (до 441 случая) [8, 9,10, 11, 12]. 
Психотические осложнения, развивающиеся на фоне употребления современных синтетических ПАВ, обусловливают различия в их клинико-диагностической оценке у врачей психиатров и психиатров-наркологов. Неоднородные данные разноплановых исследований показывают скоротечное развитие зависимости у потребителей наркотических веществ синтетического происхождения. Потребление синтетических ПАВ характеризуется стремительным переходом от периодического однократного употребления вещества к многократному в течение дня. При этом отмечается рост толерантности с увеличением доз и частоты приема - при опросе и сборе анамнеза больные сообщают о потреблении вначале «части пакетика» с ПАВ, а в дальнейшем уже о приеме «нескольких пакетиков» в день. Т.е. зависимость формируется в ограниченные по времени сроки от нескольких месяцев до года.

У потребителей данных наркотических веществ отмечается высокая частота развития психотических осложнений. При этом нужно указать, что психозы имеют особенность развиваться даже при однократном употреблении вещества. Схожесть клинической картины психозов с симптомами шизофрении создает определенные сложности в диагностике и соответствующей терапии расстройств. Психотические осложнения на фоне потребления синтетических ПАВ характеризуются психомоторным возбуждением, наличием истинных зрительных галлюцинаций и слуховых галлюцинаций; больные высказывают бредовые идеи преследования, слежки, прослушивания, намерений их убить со стороны «преследователей». Дополнительные трудности в анализе клинических проявлений этой группы психотических расстройств обусловлены особенностями определения наличия ПАВ в биологических средах организма, когда феномены могут сохраняться за рамками непосредственного действия ПАВ, что отличает подобные состояния от классических интоксикационных психозов $[13,14,15,16,17$, $18,19,20,21,22]$.

Изучение специфических особенностей и динамики клинической картины психотических расстройств, связанных с потреблением новых синтетических ПАВ, позволит решить проблему их этиологической диагностики с привлечением психометрических методов и данных комплексного лабораторного обследования.

\section{ЦЕЛЬ ИССЛЕДОВАНИЯ}

Изучить особенности клинической картины психотических расстройств, связанных с употреблением синтетических психоактивных веществ, в зависимости от длительности психотического периода и определяемого вещества в биологических жидкостях.

\section{МАТЕРИАЛЫ И МЕТОДЫ}

Клинико-психопатологическим методом обследованы 318 мужчин (средний возраст составил $35,7 \pm 7,4$ года) с психотическими расстройствами на фоне употребления современных синтетических ПАВ (так называемые соль, шоколад, спайс). Все они были госпитализированы и прошли лечение в стационаре ККБУЗ «Красноярский краевой наркологический диспансер № 1». Диагноз устанавливался согласно критериям МКБ-10 [23]. Всем испытуемым было проведено химикотоксикологическое исследование (ХТИ) на наличие наркотиков в моче на момент госпитализации. Определение вещества в моче пациентов проводилось методом количественной газожидкостной хроматографии. Пациенты с алкогольными психозами и синдромом отмены алкоголя (алкогольный абстинентный синдром) не включались в исследовательскую выборку.

\section{РЕЗУЛЬТАТЫ И ОБСУЖДЕНИЕ}

По длительности наблюдавшихся психотических расстройств все пациенты были разделены на три группы, условно обозначенные нами как I, II, III группы (рис. 1).

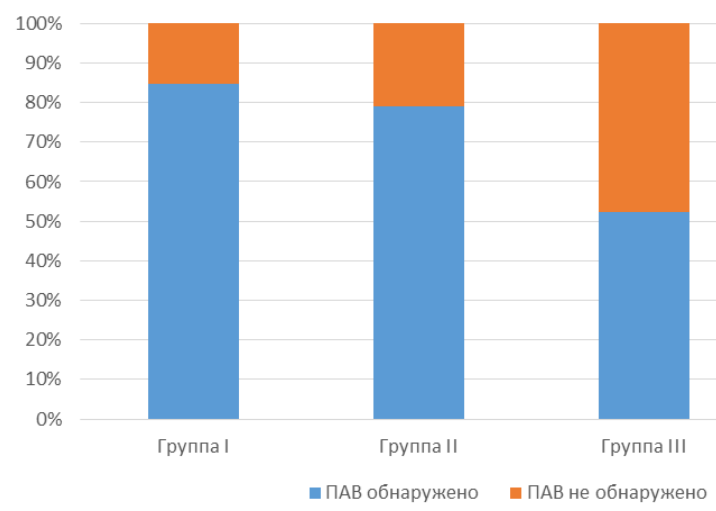

Р и с у н о к 1. Распределение пациентов с психотическими расстройствами в зависимости от длительности психотического эпизода и наличия психоактивного вещества в биологических средах организма 
Группа I (длительность психотических расстройств 1-7 суток). Данную группу составили 78 пациентов $(24,5 \%)$ с диагнозом: острая интоксикация другими стимуляторами с делирием (F15.03); психотическое расстройство, связанное с употреблением других стимуляторов, преимущественно галлюцинаторное (F15.52); психотическое расстройство, связанное с употреблением других стимуляторов, преимущественно бредовое (F15.51). В биологических жидкостях организма пациентов этой группы употребление ПАВ выявлено в $84,6 \%$ случаев $\quad(\mathrm{n}=66):$ производные пировалерона $(94,4 \%)$, каннабиноиды $(5,6 \%)$, у 12 пациентов $(15,4 \%)$ употребление ПАВ не обнаружено.

Группа II (длительность психотических расстройств 8-14 суток) сформирована из пациентов с диагнозом: острая интоксикация другими стимуляторами с делирием (F15.03); психотическое расстройство, связанное с употреблением других стимуляторов, преимущественно бредовое (F15.51); психотическое расстройство, связанное с употреблением других стимуляторов, преимущественно галлюцинаторное (F15.52); психотическое расстройство, связанное с сочетанным употреблением наркотиков и других психоактивных веществ, полиморфное (F19.53). Такая длительность наблюдалась более чем у половины пациентов $(\mathrm{n}=196,61,6 \%)$. Употребление различных ПАВ: производные пировалерона $(72,1 \%)$, кан- набиноиды $(20,3 \%)$, сочетанное употребление $(7,6 \%)$ подтвердилось у преобладающего большинства обследованных $(\mathrm{n}=155,79,1 \%)$. Вместе с тем в 41 случае $(20,9 \%)$ употребление ПАВ не выявлено.

Группа III (длительность психотических расстройств 15 и более суток) состояла из 44 больных $(13,9 \%)$ с диагнозом: психотическое расстройство, связанное с употреблением других стимуляторов, преимущественно бредовое (F15.51); психотическое расстройство, связанное с употреблением других стимуляторов, полиморфное (F15.53); психотическое расстройство, связанное с сочетанным употреблением наркотиков и других психоактивных веществ, шизофреноподобное (F19.50); психотическое расстройство, связанное с сочетанным употреблением наркотиков и других психоактивных веществ, преимущественно галлюцинаторное (F19.52). В биологических жидкостях организма употребление ПАВ имело место у $52,2 \%$ обследованных: производные пировалерона $(68,8 \%)$, каннабиноиды (12,5\%), сочетанное употребление (18,7\%). В то же время у 47,8\% пациентов применение ПАВ не доказано данными ХТИ.

В клинической картине и динамике развития психотических расстройств у больных трех выделенных групп были выявлены определенные различия (рис. 2):

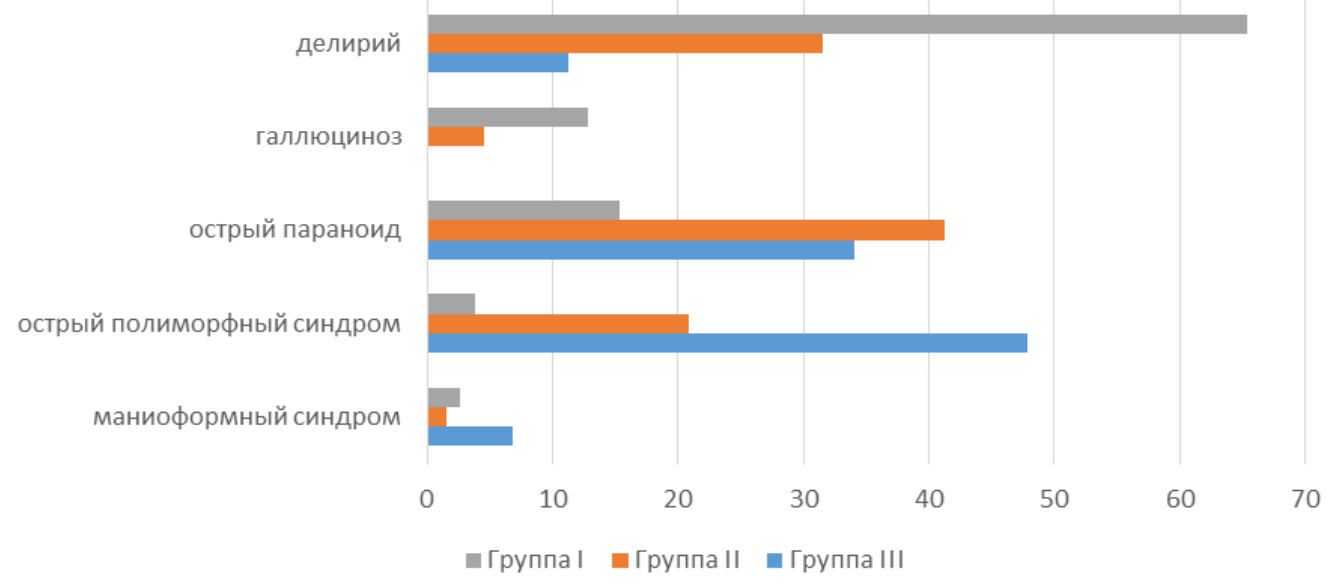

\section{Рисунок 2. Клинико-синдромологическая структура психотических расстройств у обследованных пациентов}

Очевидно, что большой удельный вес психотических расстройств в виде делирия наблюдался в группе больных с длительностью психоза 1-7 суток (группа I). В группе II, напротив, данные психотические расстройства отмечались почти в 6 раз реже. Психотическая симптоматика, наблюдающаяся у больных группы II с длительностью психоза 8-14 суток, преимущественно представлена острым параноидом и делирием, в значительно меньшей степени отмечались острый по- лиморфный синдром и галлюциноз. В группе III психотические расстройства у больных в основном были представлены в виде острого полиморфного синдрома и острого параноида, галлюциноза в этой группе больных не отмечалось.

Клинико-психопатологическая структура психотических осложнений, развившихся на фоне потребления синтетических ПАВ у пациентов, входящих в группу с длительностью психотических осложнений 1-7 суток (I группа), более чем 
в половине случаев $(65,4 \%)$ представлена делириозным синдромом. В данной группе больным были выставлены диагноз (по критериям МКБ-10):

F15.03 Острая интоксикация другими стимуляторами с делирием.

F15.51 Психотическое расстройство, связанное с употреблением других стимуляторов, преимущественно бредовое.

F15.52 Психотическое расстройство, связанное с употреблением других стимуляторов, преимущественно галлюцинаторное.

Делириозный синдром у пациентов этой группы сопровождался психомоторным возбуждением и двигательным беспокойством. В клинической картине отмечались зрительные галлюцинации угрожающего характера. Пациенты активно искали и просили помощи и поддержки у окружающих. По выходе из делирия лишь частично сохранялись воспоминания о своих болезненных переживаниях.

Пациентам, входящим в группы II и III, были выставлены следующие диагнозы в зависимости от психопатологической симптоматики:

F15.03 Острая интоксикация другими стимуляторами с делирием.

F15.51 Психотическое расстройство, связанное с употреблением других стимуляторов, преимущественно бредовое.

F15.52 Психотическое расстройство, связанное с употреблением других стимуляторов, преимущественно галлюцинаторное.

F15.53 Психотическое расстройство, связанное c употреблением других стимуляторов, полиморфное.

F19.50 Психотическое расстройство, связанное с сочетанным употреблением наркотиков и других психоактивных веществ, шизофреноподобное.

F19.52 Психотическое расстройство, связанное с сочетанным употреблением наркотиков и других психоактивных веществ, преимущественно галлюцинаторное.

F19.53 Психотическое расстройство, связанное с сочетанным употреблением наркотиков и других психоактивных веществ, полиморфное.

В группе больных с длительностью психоза 814 суток (группа II) психотические осложнения также представлены в большей степени делириозным синдромом. Наряду с психомоторным возбуждением, двигательным беспокойством в виде бесцельных движений у этих пациентов отмечалась немотивированная агрессия к окружающим, они практически не вступали в контакт, прятались и громко кричали. По выходе из делирия больные, так же как и пациенты группы I, фрагментарно амнезировали происходившие события и болезненные воспоминания.
Клиническая картина делирия у пациентов группы III (длительность психотических расстройств 15 суток и более) отличается менее агрессивным и беспокойно-возбужденным поведением. При наличии страха, бессонницы, зрительных галлюцинаций угрожающего характера, чувства слежки и преследования пациенты не просят активно помощи, не кричат, зачастую разговаривают с мнимым собеседником, пьют из несуществующего стакана и т.д. По окончании психоза лишь частично помнят о перенесенных страхах и тревожных переживаниях. Наличие страха угрозы, уверенности в опасности для жизни, идей преследования и слежки через камеры и бытовые приборы сближает картину психоза с параноидными и шизофреноформными синдромами.

При галлюцинозе клиническая картина психотических расстройств характеризовалась слуховыми галлюцинациями - от единичных акоазмов до развернутого диалога на фоне сохраняющегося сознания с правильной ориентировкой в месте, времени и собственной личности. Содержание обманов восприятия имело императивный, осуждающий, угрожающий характер, как правило, связанный с ситуацией употребления наркотиков. Больные могли слышать «голоса» друзей, которые разговаривают между собой, зовут прогуляться на улицу, угрожают расправой, сами они могли разговаривать с вымышленным собеседником по мнимому телефону, получая предупреждения о «слежке из-за наркотиков». В группах больных II и III чаще наблюдалось нанесение самоповреждений в области шеи и предплечий, отмечались тактильные галлюцинации, сенестопатии с неприятными ощущениями во внутренних органах. При синдроме тактильного галлюциноза они испытывали ощущение, что по коже ползают «блохи», «внутренности выпадают через брюшную стенку, выходят кусками» (при этом пациенты заматывались пеленками, фольгой, стараясь удержать органы внутри). Отмечались моторное беспокойство, суетливость и аффект тревоги.

Острый параноид у больных всех групп характеризовался развитием на фоне сохранного сознания бредовых идей преследования с ошибочной оценкой реальной обстановки и бескомпромиссной уверенностью в непосредственной опасности своему физическому существованию. Пациенты испытывали выраженные страх и тревогу, заявляли, что за ними следят «подозрительные люди» из окон, проезжающих машин, вмонтированных в окружающие предметы «жучков и чипов». Психомоторное возбуждение носило характер агрессивной защиты. Следует отметить, что у пациентов с длительностью психотических осложнений 1-7 суток (группа I) болезненные переживания были более аффективно окрашены. 
Острый полиморфный синдром наблюдался в половине случаев у пациентов, находящихся на лечении в стационаре более 15 суток. Симптомы различных видов известных психических состояний разной интенсивности достаточно быстро сменяли друг друга, не позволяя диагностировать ни одно из них. У больных наблюдалось состояние эйфории, отмечались явления дереализации, дурашливость в поведении с беспричинным смехом. Пациентам мир казался «ярче», все виделось в «розовом цвете», отмечались разные виды галлюцинаций - больные ощущали прикосновения и запахи, слышали звуки и голоса. Далее эйфория поэтапно сменялась тревогой и страхом, появлялись мысли, что следят «сотрудники ФСБ», чтобы «арестовать за наркотики», дурашливость переходила в психомоторное возбуждение. Наряду с симптомами, которые развились и являются общими для всех клинических групп пациентов, имеются некоторые отличия.

У больных группы I в большей степени отмечались бредовые идеи преследования, вмонтированных датчиков. В клинической картине психотических осложнений у больных группы II превалировали бредовые идеи религиозного содержания, стремление спастись от преследователей, выпрыгивая из окон и с балконов, убегая от мнимой погони раздетыми. В группе III у пациентов отмечалась кататоническая симптоматика, больные непродолжительно застывали в не меняющемся положении, занимали вычурные позы.

Маниоформный синдром характеризовался повышением настроения, ускорением мышления по темпу, ощущением «избытка энергии», подвижностью до суетливости, неадекватностью поведения реальной ситуации, непродуктивной «творческой и креативной» активностью. Больные высказывали бредовые идеи экспансивного характера с переоценкой своих возможностей, что инициировало опасные для жизни поступки (выпрыгивали на ходу из транспорта). Кроме того, высказывали идеи богатства, высоких связей, особого влияния на события, были дурашливы в поведении, гримасничали. Такая клиническая картина наблюдалась у больных всех групп.

\section{ЗАКЛЮЧЕНИЕ}

По результатам проведенного исследования можно сделать следующие выводы:

Психопатологические синдромы реакций экзогенно-токсического типа в форме вербального галлюциноза и делирия преобладают в группе пациентов с психотическими осложнениями, протекавшими с наименьшей длительностью (до 7 суток). В этой же группе отмечается наибольшая частота выявления приема ПАВ в биологических средах организма.
В группе пациентов с психотическими расстройствами с длительностью течения от 8 до 14 суток практически 2/3 психотических осложнений представлены шизофреноподобными нарушениями в форме острого параноида и острого полиморфного синдрома. При этом наличие ПАВ в биологических жидкостях было обнаружено в $79,1 \%$ случаев.

В группе пациентов с психотическими расстройствами продолжительностью 15 дней и более синдромы, характерные для классических вариантов интоксикационных психозов, практически отсутствуют. Клиническая картина психотических осложнений представлена шизофреноформными нарушениями. Анамнестические данные употребления ПАВ у 2/3 больных не были подтверждены результатами лабораторного исследования.

Клиническая картина психозов, вызванных употреблением синтетических ПАВ, значительно отличается от классической картины металкогольных психозов или реакций экзогеннотоксического типа большей длительностью течения и снижением вероятности определения вещества в биологических средах организма. Психотические расстройства, возникающие при употреблении современных синтетических ПАВ, вероятнее всего являются следствием высокой токсикокинетической и психодинамической составляющей действия данных веществ, что необходимо учитывать в диагностике и терапии психотических расстройств, развившихся на фоне их потребления.

Результаты проведенного исследования возможно использовать для разработки, создания и внедрения в практическое здравоохранение метода, позволяющего врачам психиатрам и наркологам верно интерпретировать результаты обследований пациентов с психотическими осложнениями на фоне потребления новых синтетических ПАВ.

\section{КОНФЛИКТ ИНТЕРЕСОВ}

Авторы заявляют об отсутствии явных и потенциальных конфликтов интересов в связи с публикацией данной статьи.

\section{ИСТОЧНИК ФИНАНСИРОВАНИЯ}

Авторы заявляют об отсутствии спонсорского финансирования при проведении исследования.

\section{СООТВЕТСТВИЕ ПРИНЦИПАМ ЭТИКИ}

Работа соответствует этическим стандартам Хельсинской декларации ВМА (протокол заседания этического комитета ГБОУ ВПО «Красноярский государственный медицинский университет им. проф. В.Ф. Войно-Ясенецкого» Минздрава России № 76/2016 от 04 мая 2017 г.). 


\section{ЛИТЕРАТУРA/REFERENCES}

1. Доклад Международного комитета по контролю над наркотиками за 2020 год. Вена : Управление ООН по наркотикам и преступности, 2021. 166 c. Report of the International Narcotics Control Board for 2020. Vienna: United Nations Office on Drugs and Crime, 2021:166.

2. Сизоненко А.Ю. Современные причины развития наркомании в России. Вестник науки и образования. 2019. № 18 (72). С. 70-73. Sizonenko $\mathrm{AYu}$. Modern reasons for development of drug addiction in Russia. Bulletin of Science and Education. 2019;18(72):70-73 (in Russian).

3. Михайлов Б.П., Тузов Л.Л. Актуальные проблемы профилактики наркомании и незаконного оборота наркотиков в молодежной среде. Вестник Московского университета МВД России. 2017. № 6. C. 165-169. Mikhailov BP, Tuzov LL. Relevant problems of prevention of drug addiction and drug trafficking among young people. Bulletin of the Moscow University of the Ministry of Internal Affairs of Russia. 2017;6:165-169. (in Russian).

4. Калачев Б.Ф., Сухачевская М.В., Целинский Б.Л. Подпольное производство наркотиков. Теоретические и правовые основы борьбы с незаконным оборотом наркотиков: сборник научных трудов ВНИИ МВД России. М., 1998. С. 8694. Kalachev BF, Sukhachevskaya MV, Tselinsky BL. Clandestine drug production. Theoretical and legal foundations of combating illicit drug trafficking: book of scientific works of the All-Russian Research Institute of the Ministry of Internal Affairs of Russia. M., 1998:86-94 (in Russian).

5. Головко А.И., Башарин В.А., Иванов М.Б., Баринов В.А., Бонитенко Е.Ю. Дизайнерские наркотики. Классификация, механизмы токсичности. Наркология. 2015. № 8. С. 69-85. Golovko AI, Basharin VA, Ivanov MB, Barinov VA, Bonitenko EYu. Designer drugs. Classification, mechanisms of toxicity. Narcology. 2015;8:69-85 (in Russian).

6. Доклад о наркоситуации в Российской Федерации в 2019 году. М. : Государственный антинаркотический комитет, 2020. 51 с. Report on the drug situation in the Russian Federation in 2019. Moscow: State Anti-Drug Committee, 2020:51 (in Russian).

7. Степанов И.В., Грачев Ю.А., Сагайдак А.Ю. Наркоситуация в Российской Федерации, основные тенденции ее развития. Вестник СанктПетербургского МВД России. 2016. № 2 (70). C. 126-129. Stepanov IV, Grachev YuA, Sagaidak $\mathrm{AYu}$. The drug situation in the Russian Federation, the main trends of its development. Bulletin of the St. Petersburg Ministry of Internal Affairs of Russia. 2016;2(70):126-129 (in Russian).

8. Киржанова В.В., Киржанов В.Н., Григорова Н.И., Сидорюк О.В. Состояние и деятельность наркологической службы в Российской Федерации в 2017 году. Аналитический обзор. М. :
ФГБУ «НМИЦ ПН им. В.П. Сербского» Минздрава России, 2019. 196 с. Kirzhanova VV, Kirzhanov VN, Grigorova NI, Sidoryuk OV. The state and activity of the narcological service in the Russian Federation in 2017. Analytical review. Moscow: FSBI "V.P. Serbsky National Medical Research Center of Psychiatry and Narcology" of the Ministry of Health of Russia, 2019. 196 p. (in Russian).

9. Пичугина Ю.А., Березовская М.А., Коробицина Т.В. Анализ современной ситуации употребления психоактивных веществ. Сибирское медицинское обозрение. 2016. № 1 (97). С. 40-47. Pichugina YuA, Berezovskaya MA, Korobitsina TV. Analysis of the current situation of psychoactive substances use. Siberian Medical Review. 2016;1(97):40-47 (in Russian).

10. Гапонов С.В. Актуальные вопросы мониторинга наркоситуации в Красноярском крае. Вестник Сибирского юридического института ФСКН России. 2013. № 5. С. 15-18. Gaponov SV. Relevant issues of monitoring the drug situation in the Krasnoyarsk Territory. Bulletin of the Siberian Law Institute of the Federal Drug Control Service of Russia. 2013;5:15-18 (in Russian).

11. Доклад о наркоситуации в Красноярском крае в 2019 году. [Электронный ресурс]. Report on the drug situation in the Krasnoyarsk Territory in 2019. [Electronic resource] (in Russian). http://www.krskstate.ru/safety/ank/info

12. Чухрова М.Г., Пронин С.В., Рыбальчук Н.В., Иванова В.Э. Психические и психосоматические последствия потребления спайсов. Мир науки, культуры, образования. 2015. № 1 (50). C. 423-426. Chukhrova MG, Pronin SV, Rybalchuk NV, Ivanova VE. Mental and psychosomatic consequences of spice use. World of Science, Culture, Education. 2015;1(50):423-426 (in Russian).

13. Хорошилов Г.П., Худяков А.В. Интоксикационные психозы, связанные с употреблением современных психоактивных веществ. Практическая медицина. 2015. № 5 (90). С. 7-10. Khoroshilov GP, Khudyakov AV. Intoxication psychoses associated with the use of modern psychoactive substances. Practical Medicine. 2015;5(90):7-10 (in Russian).

14. Бохан Н.А., Селиванов Г.Ю. Клиническая типология психопатологических расстройств у потребителей синтетических каннабиноидов (спайсов). Сибирский вестник психиатрии и наркологии. 2015. № 4 (89). С. 18-23. Bokhan NA, Selivanov GYu. Nosological characteristics of states of dependence on synthetic cannabinoids ("Spice"). Siberian Herald of Psychiatry and Addiction Psychiatry. 2015;4(89):18-23 (in Russian).

15. Бохан Н.А., Селиванов Г.Ю., Блонский К.А. Характеристика абстинентного синдрома у лиц, страдающих зависимостью от употребления синтетических каннабиноидов (спайсов). Сибирский вестник психиатрии и наркологии. 
2016. № 4 (93). C. 45-50. Bokhan NA, Selivanov $\mathrm{GYu}$, Blonsky KA. Characteristics of withdrawal symptoms in people addicted to the use of synthetic cannabinoids ("Spice"). Siberian Herald of Psychiatry and Addiction Psychiatry. 2014;4(93):18-23 (in Russian).

16. Афанасьева Н.А., Березовская М.А., Коробицина Т.В. и др. Клинико-динамические особенности интоксикационных психозов, вызванных употреблением современных синтетических психоактивных веществ. Сибирский вестник психиатрии и наркологии. 2017. № 3 (96). С. 7883. Afanasyeva NA, Berezovskaya MA, Korobitsina TV, Pichugina YuA, Sergienko NN. Clinical and dynamic features of intoxication psychoses due to the use of modern synthetic psychoactive substances. Siberian Herald of Psychiatry and Addiction Psychiatry. 2017;3(96):78-83 (in Russian). doi: 10.26617/1810-3111-2017-3(96)-78-83

17. Дубатова И.В., Стоякин И.В., Карнаух К.А., Сафроненко А.В. Клиническая значимость и особенность психических расстройств у потребителей «дизайнерских» наркотиков. Уральский медицинский журнал. 2017. № 5 (149). С. 104108. Dubatova IV, Stoyakin IV, Karnaukh KA, Safronenko AV. Clinical significance and specificity of mental disorders in "designer" drug users. Ural Medical Journal. 2017;5(149):104-108 (in Russian).

18. Патрикеева О.Н., Овчинников А.А., Кормилина О.М. Интоксикационные психозы у потребителей синтетических каннабиноидов. Наркология. 2015. T. 14, № 1. C. 41-44. Patrikeeva ON, Ovchinnikov AA, Kormilina OM. Intoxication psychoses in users of synthetic cannabinoids. Narcology. 2015;14,1:41-44 (in Russian).

19. Клименко Т.В., Шахова С.М., Козлов А.А. Психотические расстройства вследствие употребления синтетических каннабиноидов (спайсов). Сибирский вестник психиатрии и наркологии. 2017. № 2 (95). C. 26-30. Klimenko TV, Shakhova
SM, Kozlov AA. Psychotic disorders after use of synthetic cannabinoids (spice). Siberian Herald of Psychiatry and Addiction Psychiatry. 2017;2(95):26-30 (in Russian).

20. Anizan S, Concheiro M, Lehner KR, Bukhari MO, Suzuki M, Rice KC, Baumann MH, Huestis MA. Linear pharmacokinetics of 3,4methylenedioxypyrovalerone (MDPV) and its metabolites in the rat: relationship to pharmacodynamic effects. Addict Biol. 2016 Mar;21(2):339-47. doi: 10.1111/adb.12201. Epub 2014 Dec 5. PMID: 25475011; PMCID: PMC5362107.

21. Beck O, Franzen L, Backberg M. Toxicity evaluation of -pyrrolidinovalerophenone (-PVP): results from intoxication cases within the STRIDA project. Clinical Toxycol (Phila). 2016;54(7):568-575.

22. Ракитин С.А., Курушкин М.В., Мищенко С.В., Зубкова Т.А. Клинический пример психоза, развившегося на фоне острой интоксикации синтетическими каннабиноидами. Омский психиатрический журнал. 2020. № 2 (25). C. 8-11 Rakitin SA, Kurushkin MV, Mishchenko SV, Zubkova TA. Clinical example of psychosis developed against the background of acute intoxication with synthetic cannabinoids. Omsk Psychiatric Journal. 2020;2(25):8-11 (in Russian). doi: 10.24411/24128805-2020-10206

23. Чуркин А.А., Мартюшов А.Н. Практическое руководство по использованию МКБ-10 в психиатрии и наркологии. М. : Издательство ГНЦ СиСП им. В.П. Сербского, 2004. 140 с. Churkin AA, Martyushov AN. A practical guide to the use of ICD-10 in psychiatry and narcology. Moscow: Publishing House of V.P. Serbsky State Scientific Center for Social and Forensic Psychiatry, 2004:140 (in Russian).

Поступила в редакцию 24.03.2021

Утверждена к печати 28.05.2021

Афанасьева Наталья Анатольевна - ассистент кафедры психиатрии и наркологии с курсом ПO, ORCID iD 0000-0002-5630-6709.

Коробицина Татьяна Валерьевна - д-р мед. наук, профессор кафедры психиатрии и наркологии с курсом ПО, профессор кафедры теории и методики социальной работы. AuthorID 696459. ResearcherID L-5601-2018. tvkor@mail.ru

Пичугина Юлия Анатольевн - к.м.н., доцент кафедры психиатрии и наркологии с курсом ПО, AuthorID 185358. ORCID iD 0000-0001-8391-821X. yulia651@ mail.ru.

Юков Олег Сергеевич - ассистент кафедры психиатрии и наркологии с курсом ПО, врач-психиатр. KafedraPN@mail.ru

Ширшова Алина Александровна - студентка 6-го курса лечебного факультета.

\section{Афанасьева Наталья Анатольевна, afanaseva76@yahoo.com}


For citation: Afanasyeva N.A., Korobitsina T.V., Pichugina Yu.A., Yukov O.S., Shirshova A.A. Clinical and syndromological features of intoxication psychoses due to the use of modern synthetic psychoactive substances. Siberian Herald of Psychiatry and Addiction Psychiatry. 2021; 2 (111): 46-53. https://doi.org/10.26617/1810-3111-20212(111)-46-53

\title{
Clinical and syndromological features of intoxication psychoses due to the use of modern synthetic psychoactive substances

\author{
Afanasyeva N.A. ${ }^{1}$, Korobitsina T.V. ${ }^{1,}$, Pichugina Yu.A. ${ }^{1}$, Yukov O.S. ${ }^{1}$,
} Shirshova A.A. ${ }^{1}$
}

\author{
${ }^{1}$ Krasnoyarsk State Medical University named after Professor V.F. Voyno-Yasenetsky \\ Partizan Zheleznyak Street 1, 660022, Krasnoyarsk, Russian Federation \\ ${ }^{2}$ Siberian Federal University \\ Svobodnyy Avenue 79, 660041, Krasnoyarsk, Russian Federation
}

\begin{abstract}
Relevance. The continuous production of newly synthesized analogs of synthetic drugs with their immediate delivery to the drug market reflects the growing trend in the abuse of substances under international control. A comprehensive analysis of the clinical picture of intoxication psychoses and the medico-social consequences of the use of modern synthetic psychoactive substances will make it possible to develop therapeutic approaches to the treatment and rehabilitation of such patients. Objective. To study the features of psychoses that developed against the background of the use of synthetic psychoactive substances. Research Material. Three groups of patients were formed (318 male patients in total, mean age $35.7 \pm 7.4$ years) in accordance with the duration of the psychotic period. Methods: clinical and psychopathological, anamnestic, follow-up, gas-liquid chromatography. Results. Data were obtained on the features of the clinical picture of psychotic complications depending on the duration of psychosis, as well as on the identification of a psychoactive substance in the biological fluids of the patient's body. Conclusion. The clinical and syndromological features of psychotic disorders that developed against the background of the use of new synthetic psychostimulants and cannabinoids were determined. With practically the same symptom complex in the clinical picture of such psychoses, certain differences were noted taking into account the duration of psychotic complications.
\end{abstract}

Keywords: intoxication psychoses, psychoactive substances, schizophreniform mental disorders, duration of psychotic period.

Received March 24.2021

Accepted May 28.2021

Afanasyeva Natalya A. - assistant of the Department of Psychiatry and Narcology with a vocational education course, Krasnoyarsk State Medical University named after Professor V.F. Voyno-Yasenetsky, Krasnoyarsk, Russian Federation. ORCID iD 0000-0002-5630-6709.

Korobitsina Tatiana V. - MD, Professor of the Department of Psychiatry and Narcology with a vocational education course, Krasnoyarsk State Medical University named after Professor V.F. Voyno-Yasenetsky, Krasnoyarsk, Russian Federation; Professor of the Department of the Theory and Methodology of Social Work, Siberian Federal University, Krasnoyarsk, Russian Federation. AuthorID 696459. ResearcherID L-5601-2018. tvkor@mail.ru

Pichugina Yuliya A. - PhD, Associate Professor of the Department of Psychiatry and Narcology with a vocational education course, Krasnoyarsk State Medical University named after Professor V.F. Voyno-Yasenetsky, Krasnoyarsk, Russian Federation. ORCID iD 0000-0001-8391-821X. AuthorID 185358. yulia651@mail.ru.

Yukov Oleg S. - assistant of the Department of Psychiatry and Narcology with a vocational education course, psychiatrist, Krasnoyarsk State Medical University named after Professor V.F. Voyno-Yasenetsky, Krasnoyarsk, Russian Federation. KafedraPN@mail.ru

Shirshova Alina A. - 6th year student of the Medical Faculty, Krasnoyarsk State Medical University named after Professor V.F. Voyno-Yasenetsky, Krasnoyarsk, Russian Federation

Afanasyeva Natalya A., afanaseva76@yahoo.com 